\title{
BMJ Open Functional lesional neurosurgery for tremor-a protocol for a systematic review and meta-analysis
}

\author{
Sebastian R Schreglmann, ${ }^{1}$ Joachim K Krauss, ${ }^{2}$ Jin Woo Chang, ${ }^{3}$ \\ Kailash P Bhatia, ${ }^{1}$ Georg Kägi ${ }^{4}$
}

To cite: Schreglmann SR, Krauss JK, Chang JW, et al. Functional lesional neurosurgery for tremor-a protocol for a systematic review and meta-analysis. BMJ Open 2017;7:e015409. doi:10.1136/ bmjopen-2016-015409

- Prepublication history and additional material are available. To view, these files please visit the journal online (http://dx.doi.org/ 10.1136/ bmjopen-2016-015409)

Received 5 December 2016 Revised 24 March 2017 Accepted 4 April 2017

CrossMark

${ }^{1}$ Department of Motor Neuroscience and Movement Disorders, Institute of Neurology, London, UK

${ }^{2}$ Department of Neurosurgery, Medizinische Hochschule Hannover, Hannover, Germany ${ }^{3}$ Department of Neurosurgery, Yonsei University College of

Medicine, Seodaemun-gu,

Seoul, South Korea

${ }^{4}$ Department of Neurology, Kantonsspital St. Gallen, St. Gallen, Switzerland

Correspondence to Dr Sebastian R SchregImann; skgtsrs@ucl.ac.uk

\section{ABSTRACT}

Introduction The recent introduction of incision-less lesional neurosurgery using Gamma Knife and MRIguided focused ultrasound has revived interest in lesional treatment options for tremor disorders. Preliminary literature researches reveal that the consistency of treatment effects after lesional neurosurgery for tremor has not formally been assessed yet. Similarly, the efficacy of different targets for lesional treatment and incidence of persistent side effects of lesional neurosurgical interventions has not been comprehensively assessed. This work therefore aims to describe a suitable process how to review the existing literature on efficacy and persistent side effects of lesional neurosurgical treatment for tremor due to Parkinson's disease, essential tremor, multiple sclerosis and midbrain/rubral tremor.

Methods and analysis We will search electronic databases (Medline, Cochrane) and reference lists of included articles for studies reporting lesional interventions for tremor in cohorts homogeneous for tremor aetiology and intervention (technique and target). We will include cohorts with a minimum number of five subjects and follow-up of 2 months. One investigator will perform the initial literature search and two investigators then independently decide which references to include for final efficacy and safety analysis. After settling of disagreement, data will be extracted from articles using a standardised template. We will perform a random-effect meta-analysis calculating standardised mean differences (Hedge's g) for comparison in Forest plots and subgroup analysis after assessment of heterogeneity using $\mathrm{I}^{2}$ statistics.

Ethics and dissemination This study will summarise the available evidence on the efficacy of lesional interventions for the most frequent tremor disorders, as well as for the incidence rate of persisting side effects after unilateral lesional treatment. This data will be useful to guide future work on incision-less lesional interventions for tremor.

Systematic review registration This study has been registered with the PROSPERO database (no. CRD42016048049).

\section{INTRODUCTION}

Tremor is defined as an involuntary, oscillating sinusoidal movement of a body part and is a frequent symptom in Parkinson's disease (PD), essential tremor (ET) and in multiple sclerosis (MS) or after midbrain
Strengths and limitations of this study:

Protocol for a formal, systematic review and metaanalysis of lesional functional neurosurgery for tremor.

- Comprehensive comparison of consistency and efficacy of lesional targets in most prevalent tremor aetiologies.

- First meta-analysis of persistent side effect prevalence after lesional neurosurgical treatment.

- Protocol to establish safety and efficacy benchmarks for emerging incision-less lesional functional neurosurgery approaches.

- Frequent retrospective nature and potential reporting bias of primary source data will be addressed.

lesions. ET is one of the most common movement disorders affecting up to $4.6 \%$ of the population $\geq 65$ years. ${ }^{1}$ While most patients do well with first-line oral medication like beta-blockers or primidone, at least $50 \%$ of them do not tolerate this long term, ${ }^{2}$ leaving $10 \%$ severely disabled by their tremor, losing dexterity to a great extent. ${ }^{3}$ Similarly in PD, tremor is one of the most challenging symptoms to treat with oral medication ${ }^{4}$ and this group of severely incapacitated patients depends on advanced therapeutic options. ${ }^{5}$

Although its phenomenology considerably differs between the above-mentioned aetiologies, our current understanding points at a common abnormal central oscillatory activity within a network involving motor cortex, thalamus, globus pallidum and cerebellum..$^{6-8}$ Accordingly, lesional surgical interventions within parts of this network using functional neurosurgery have been used successfully since the 1940s. ${ }^{9}$ Over time, interventions at various anatomical structures within this network have been studied ${ }^{9-12}$ and it is now generally accepted that thalamotomy, influencing afferent cerebellar signalling, provides the highest level of tremor symptom relief. 
During the past two decades, lesional interventions, although performed worldwide, ${ }^{13}$ were largely superseded in the academic setting by stimulation technology ${ }^{14}$ which contributed dramatically to our understanding of tremor pathoaetiology. ${ }^{15-18}$ Since the introduction of Gamma Knife (GK), incision-less functional neurosurgery, that is, lesion placement through the intact skull, is a possibility ${ }^{12}$ and the recent addition of MRI-guided high-intensity focused ultrasound (MRIgFUS) ${ }^{19-22}$ has again stimulated interest in this field. ${ }^{23} 24$

A preliminary literature search performed in June 2016 suggests a wealth of studies on this topic, although the majority of published reports are of small or medium size. Obvious heterogeneity in study design, data collection, documentation and presentation limit the accessibility of these data and complicate its interpretation. So far, there are no reliable estimates on the consistency of treatment effects after lesional interventions for tremor. Similarly, the prevalence of persisting side effects after such interventions has not been compared in a comprehensive way. We therefore aim to summarise the available data on lesional functional surgery for tremor disorders to allow comparisons between aetiologies, treatment targets and techniques. The limitations of earlier reports with regard to established and recognised diagnostic criteria, use of validated clinical assessment tools and electrophysiological or imaging-based target verification ${ }^{10}$ led us to restrict the literature search to a publication date from 1990 onwards.

We specifically aim to answer the following questions:

What is the efficacy of lesional neurosurgical interventions on tremor severity in tremor due to PD, ET, MS and midbrain/rubral origin for different lesioning techniques and targets according to published, peer-reviewed studies?

What is the prevalence rate of persistent side effects after unilateral lesional interventions for different lesioning technique and target according to published, peer-reviewed studies?

This will allow to objectively assess the safety and efficacy of existing lesional tremor treatment approaches and to compare novel, incision-less lesional interventions with this benchmark.

\section{METHODS AND ANALYSIS}

\section{Protocol}

The methods for this systematic review have been developed according to the recommendations from the Preferred Reporting Items for Systematic Review and Meta-Analysis Protocols (PRISMA-P) 2015 statement. ${ }^{25}$ This systematic review protocol has been registered in the International Prospective Register of Systematic reviews (PROSPERO) on 20 September 2016: CRD42016048049. A PRISMA-P file is attached (see online supplementary material 1).

\section{Eligibility criteria}

Cohorts reporting a minimum of five patients of or above the age of 18 years with a tremor diagnosis of confirmed aetiology, subjected to unilateral or bilateral lesional functional neurosurgery in a central neuroanatomical structure (thalamus, pallidum, subthalamic nucleus, alternative subcortical targets) by means of an intracerebral lesion, either by incisional (placement of a stylette, leukotome, cryosurgery or radiofrequency (RF) probe after skull opening) or incision-less (MRIgFUS, GK) means. Cases that received lesional functional neurosurgery in more than one anatomical structure at the same time or non-lesional approaches including deep brain stimulation were excluded.

\section{Outcome measures}

As we expect only limited amount of data from controlled trials to be found, this protocol aims to assess intervention effects by comparing preinterventional and postinterventional states. Primary outcome measure will be the change in upper limb tremor severity from baseline to follow-up time points, as reported on a validated tremor rating scale (United Parkinson's Disease Rating Scale, part III, Clinical Rating Scale for Tremor ${ }^{26}$ 27, Whiget Tremor Scale, etc). Results from controlled trials comparing lesional interventions to alternative interventions, for example, best medical treatment or deep brain stimulation were included and discussed in a narrative way as far as meaningful for comparison with lesional interventions. As we expect the literature to be heterogeneous in terms of follow-up duration and applied tremor rating scales, we aimed to primarily summarise the outcome as standardised mean difference (Hedge's g) ${ }^{28}$ irrespective of follow-up duration. To limit bias, we chose the follow-up time point with the largest number of patients retained in the analysis. Homogeneous cohorts (same tremor aetiology, intervention target and technique) were grouped together for subgroup analysis, if they consisted of a minimum of $\mathrm{n}=2$.

Secondary outcome measure was the frequency of reported persistent side effects after unilateral lesions per indication and intervention group, calculated as $\%$ of cases per group. In addition, we aim to calculate the mean rate of dysarthria and gait difficulties reported for unilateral versus bilateral procedures. Homogeneous cohorts (tremor aetiology, intervention target and technique) were grouped together for analysis, if they consisted of a minimum of $\mathrm{n}=2$.

\section{Study design}

The choice of inclusion/exclusion criteria reflects that we expect very few randomised trials in this field. Inclusion: randomised controlled trials (RCTs), meta-analysis, case-control, prospective and retrospective case series. Exclusion: studies reporting results from mixed aetiologies (subjects of different aetiologies grouped together) or mixed interventions (different anatomical targets grouped together). We included peer-reviewed articles 
Table 1 Planned inclusion and exclusion criteria

\begin{tabular}{|c|c|c|}
\hline & Inclusion criteria & Exclusion criteria \\
\hline Study design & $\begin{array}{l}\text { Randomised controlled trials, meta-analysis, case-control, } \\
\text { prospective and retrospective case series } \\
\text { A minimum of five subjects included per cohort (indication/ } \\
\text { treatment) } \\
\text { Minimum follow-up of } 2 \text { months after the intervention }\end{array}$ & $\begin{array}{l}\text { Studies reporting results from } \\
\text { mixed aetiologies or mixed } \\
\text { intervention (different anatomical } \\
\text { targets or techniques) }\end{array}$ \\
\hline Type of publication & Peer-reviewed articles without language restriction & Letters, abstracts and editorials \\
\hline
\end{tabular}

without language restriction. Letters, abstracts and editorials were not included (see table 1 ).

\section{Information sources and search strategy}

A full search of MEDLINE and Cochrane (Ovid) database will be performed limited to time of publication (between January 1990 and February 2017), using 'tremor*' AND 'lesion*', 'neurosurg*' ,'thalamotomy', 'subthalamotomy', 'pallidotomy' as search terms. Contact with authors was made if needed (table 2).

\section{Data collection}

Primary database searches will be performed by one researcher who will compile a list of non-duplicate studies according to inclusion and exclusion criteria. In addition to the primary searches, we will identify relevant studies from the reference lists of primary search results. From this list, two researchers independently will compile a definitive list of studies to be included in the safety and efficacy analysis-before analysis, lists will be compared and discrepancies settled. Data will be extracted from original sources by use of a standardised template. As we aim to cover publications from the past 26 years, which might cover interventions dating back to $>35$ years, we deem it unrealistic to compile data on an individual participant basis, unless given in publications.

\section{Data items}

1. Publication details: title, authors, publication year;

2. Design: proretrospective, randomisation, blindassessment, controlled;

3. Clinical details: cohort size, anatomical target, treatment technique, unilateral or bilateral intervention, guidance/targeting technique, tremor scale and item used, preinterventional and postinterventional tremor score $($ mean $\pm \mathrm{SD})$, follow-up duration, art/number and severity of transient and persistent side effects reported;

4. Quality assessment according to Jadad et $a l^{29}$ and Newcastle-Ottawa Scale. ${ }^{30}$

\section{Bias assessment}

If several follow-up time points are reported per cohort, the time point with the largest number of subjects retained will be chosen to minimise selection/reporting bias. We will assess the quality of RCTs (Jadad Scale ${ }^{29}$ )

Table 2 Search strategy

\begin{tabular}{|c|c|c|}
\hline Item & Search term & $\begin{array}{l}\text { Boolean } \\
\text { operator }\end{array}$ \\
\hline Tremor & $\begin{array}{l}\text { ('tremor }{ }^{*}[\text { All Fields] OR 'tremor"' }[\text { MeSH Terms], 'shaking' [All Fields] OR 'shaking' } \\
\text { [MeSH Terms]) }\end{array}$ & AND \\
\hline Lesion & $\begin{array}{l}\text { ('lesion*[All Fields] OR 'lesion*' [MeSH Terms], 'thalamotomy’ [All Fields] OR } \\
\text { 'thalamotomy' [MeSH Terms], 'subthalamotomy' [All Fields] OR 'subthalamotomy' } \\
\text { [MeSH Terms], 'pallidotomy' [All Fields] OR 'pallidotomy' [MeSH Terms], }\end{array}$ & AND \\
\hline Time period & Between 1 January 1990 and 1 February 2017 & \\
\hline
\end{tabular}


and non-randomised trials (Newcastle-Ottawa Scale ${ }^{30}$ ) by means of standardised assessment tools and discuss the limitations of the data synthesis in terms of study and data quality. In addition to that, in the discussion of resultsdepending on the overall quality of data-we will discuss potential shortcomings of our source data, as retrospective analyses with incomplete follow-up tend to introduce bias.

A formal assessment of publication bias however only makes sense in the presence of a sufficient number $(>10)$ of homogeneous data sets. ${ }^{31}$ As we expected the data compiled in this analysis to be of limited homogeneity, we do not plan to formally calculate bias assessments such as by means of Funnel plots, as this could result in misleading results in small and heterogeneous data sets. ${ }^{31}$ This shall only be calculated in case subgroups with $>10$ studies included are shown to have no substantial level of heterogeneity $\left(\mathrm{I}^{2}<50 \%\right)$.

\section{Data synthesis and statistics}

Aggregate data on preinterventional/postinterventional tremor severity will be extracted from publications or calculated from them in the form of mean \pm SD for outcome variables per indication/intervention group per publication. Data for continuous outcome measures will be used to calculate standardised mean difference (Hedge's g) values including $95 \% \mathrm{CIs}^{28}$ and to compute Forest plots using the Meta-Essentials workbook4 toolbox. ${ }^{32}$ Based on study heterogeneity, we will use a random-effects meta-analysis for quantitative comparison. Heterogeneity between studies will be assessed using $\mathrm{I}^{2}$ statistics, with an $\mathrm{I}^{2}>50 \%$ regarded as an indicator of substantial heterogeneity.

As we are planning to analyse data from different tremor aetiologies and interventions, we will undertake subgroup analyses by the following subgroups: PD tremor: RF ablation ventral intermedial (V.im.) nucleus, RF ablation globus pallidus internus, RF ablation subthalamic nucleus, GK ablation V.im.; ET tremor: RF ablation V.im., GK ablation V.im., MRIgFUS ablation V.im. MS tremor: RF ablation V.im., GK ablation V.im. Formal subgroup analysis was done in case of groups of a minimum of two studies per study intervention, target and aetiology. We have not planned to restrict this meta-analysis to particular targeting modalities in order not to fragment results, although we are aware that older modalities, such as ventriculography or CT-based approaches, are not used anymore at least in the Western Hemisphere academic setting.

We will provide a narrative synthesis of results structured by aetiological category and intervention type and also discuss the influence of study design and follow-up, taking Grading of Recommendations Assessment, Development and Evaluation (GRADE) guidelines into consideration. ${ }^{33}$ We will also discuss tremor recurrence in the narrative section of this review, as we expected only limited data on this in the bulk of reports.

\section{ETHICS AND DISSEMINATION}

The aim of this systematic review and meta-analysis is to summarise the data on consistency and efficacy of lesional functional neurosurgical interventions for the treatment of tremor disorders. The recent development of incision-less lesional functional neurosurgery techniques warrants this careful reassessment of the existing literature to guide future research into lesional interventions. Ethically, we consider it an obligation to summarise these data in a systematic manner to optimise treatment outcome for future patients. It will provide the basis to compare the efficacy of lesional interventions across anatomical targets, tremor aetiologies and lesional techniques. Furthermore, the calculation of prevalence rates of persistent side effects after unilateral lesional interventions will allow for safety comparisons of established, incisional lesioning techniques and novel incision-less procedures, such as MRIgFUS and GK. This will allow a more unbiased evaluation of the effects of bilateral interventions of the past and possible future.

We are committed to publish the results of this study in a peer-reviewed journal to distribute the outcome of this work. To maximise data transparency, we aim to include the data extracted from published sources in our final publication in the form of a table. This protocol, as well as it's registration and publication with the PROSPERO database (no. CRD42016048049) documents our continuing efforts of transparent research.

Acknowledgements SRS is supported by grants by the Swiss Science Foundation, the Swiss Neurological Society and the European Academy of Neurology. We would like to thank Ashwani Jha for many helpful discussions on the statistical analysis.

Contributors SRS initialised and conceptualised the research plan for the proposed systematic review and wrote the first manuscript draft. KB provided critical input on the proposed methodology and statistical analysis and reviewed the manuscript for important intellectual content. JKK and JWC reviewed the methodology and reviewed the manuscript for important intellectual content. GK supervised the project and corrected the manuscript for important intellectual content. All authors approved of the final version of the manuscript.

Funding This research received no specific grant from any funding agency in the public, commercial or not-for-profit sectors.

Competing interests None declared.

Provenance and peer review Not commissioned; externally peer reviewed.

Open Access This is an Open Access article distributed in accordance with the Creative Commons Attribution Non Commercial (CC BY-NC 4.0) license, which permits others to distribute, remix, adapt, build upon this work non-commercially, and license their derivative works on different terms, provided the original work is properly cited and the use is non-commercial. See: http://creativecommons.org/ licenses/by-nc/4.0/

C Article author(s) (or their employer(s) unless otherwise stated in the text of the article) 2017. All rights reserved. No commercial use is permitted unless otherwise expressly granted.

\section{REFERENCES}

1. Louis ED, Ferreira JJ. How common is the most common adult movement disorder? Update on the worldwide prevalence of essential tremor. Mov Disord 2010;25:534-41.

2. Lyons KE, Pahwa R. Pharmacotherapy of essential tremor : an overview of existing and upcoming agents. CNS Drugs 2008;22:1037-45.

3. Deuschl G, Raethjen J, Hellriegel $\mathrm{H}$, et al. Treatment of patients with essential tremor. Lancet Neurol 2011;10:148-61. 
4. Speelman JD, Schuurman R, de Bie RM, et al. Stereotactic neurosurgery for tremor. Mov Disord 2002;17:84-88.

5. Pahwa R, Lyons KE. Essential tremor: differential diagnosis and current therapy. Am J Med 2003;115:134-42.

6. Raethjen J, Muthuraman M. Cause or compensation? complex changes in cerebello-thalamo-cortical networks in pathological action tremor. Brain 2015;138:2808-10.

7. Helmich RC, Janssen MJ, Oyen WJ, et al. Pallidal dysfunction drives a cerebellothalamic circuit into Parkinson tremor. Ann Neurol 2011;69:269-81.

8. Cagnan $\mathrm{H}$, Little $\mathrm{S}$, Foltynie $\mathrm{T}$, et al. The nature of tremor circuits in parkinsonian and essential tremor. Brain 2014;137:3223-34.

9. Gildenberg PL, Krauss JK. 4 A-01 History of Stereotactic Surgery. In:Textbook of sterotactic and Functional Neurosurgery. J Springer, 2008:1-32.

10. Guridi J, Lozano AM. A brief history of pallidotomy. Neurosurgery 1997;41:1169-83.

11. Higuchi Y, Matsuda S, Serizawa T. Gamma knife radiosurgery in movement disorders: indications and limitations. Mov Disord $2017 ; 32$.

12. Campbell AM, Glover J, Chiang VL, et al. Gamma knife stereotactic radiosurgical thalamotomy for intractable tremor: a systematic review of the literature. Radiother Oncol 2015;114:296-301.

13. Jourdain VA, Schechtmann G. Health economics and surgical treatment for Parkinson's disease in a world perspective: results from an international survey. Stereotact Funct Neurosurg 2014;92:71-9.

14. Benabid AL, Chabardes S, Mitrofanis J, et al. Deep brain stimulation of the subthalamic nucleus for the treatment of Parkinson's disease. Lancet Neurol 2009;8:67-81.

15. Kitagawa M, Murata J, Kikuchi S, et al. Deep brain stimulation of subthalamic area for severe proximal tremor. Neurology 2000;55:114-6.

16. Herzog J, Hamel W, Wenzelburger R, et al. Kinematic analysis of thalamic versus subthalamic neurostimulation in postural and intention tremor. Brain 2007;130:1608-25.

17. Pahwa R, Lyons KE, Wilkinson SB, et al. Comparison of thalamotomy to deep brain stimulation of the thalamus in essential tremor. Mov Disord 2001:16:140-3.

18. Cagnan H, Brittain JS, Little S, et al. Phase dependent modulation of tremor amplitude in essential tremor through thalamic stimulation. Brain 2013;136:3062-75.
19. Martin E, Jeanmonod D, Morel A, et al. High-intensity focused ultrasound for noninvasive functional neurosurgery. Ann Neurol 2009;66:858-61.

20. Lipsman N, Schwartz ML, Huang Y, et al. MR-guided focused ultrasound thalamotomy for essential tremor: a proof-of-concept study. Lancet Neurol 2013;12:462-8.

21. Elias WJ, Huss D, Voss T, et al. A pilot study of focused ultrasound thalamotomy for essential tremor. N Engl J Med 2013;369:640-8.

22. Chang WS, Jung HH, Kweon EJ, et al. Unilateral magnetic resonance guided focused ultrasound thalamotomy for essential tremor: practices and clinicoradiological outcomes. $J$ Neurol Neurosurg Psychiatry 2015;86:257-64.

23. Hariz M. Focused ultrasound thalamotomy improves essential tremor. Mov Disord 2013;28:28.

24. Deuschl G. New hope for severe essential tremor? Lancet Neurol 2013;12:420-2.

25. Shamseer L, Moher D, Clarke M, et al. Preferred reporting items for systematic review and meta-analysis protocols (PRISMA-P) 2015: elaboration and explanation. BMJ 2015;349:g7647.

26. Fahn S, Tolosa E, Marin C. Clinical rating scale for tremor. In: Jankovic J, Tolosa E, eds. Parkinsons Diseae and Movement Disorders Munich1988:225-34.

27. Stacy MA, Elble RJ, Ondo WG, et al. Assessment of interrater and intrarater reliability of the Fahn-Tolosa-Marin Tremor Rating Scale in essential tremor. Mov Disord 2007;22:833-8.

28. Higgins J, Green S. The cochrane handbook for systematic reviews of interventions. In: Cochrane handbook for systematic reviews of interventions, 2011. www.handbook.cochrane.org.

29. Jadad AR, Moore RA, Carroll D, et al. Assessing the quality of reports of randomized clinical trials: is blinding necessary? Control Clin Trials 1996;17:1-12.

30. Wells G, Shea B, OConnell D. The Newcastle-Ottawa Scale (NOS) for assessing the quality if nonrandomized studies in meta-analyses. http://wwwohrica/programs/clinical epidemiology/oxford.asp.

31. Lau J, loannidis JP, Terrin N, et al. The case of the misleading funnel plot. Bmj 2006;333:597-600.

32. Van Rhee HJ, Suurmond R, Hak T. User manual for Meta-Essentials: workbooks for meta-analysis (Version 1.0), 2015. www.erim.eur. nlresearch-supportmeta-essentials

33. Guyatt GH, Oxman AD, Montori V, et al. GRADE guidelines: 5 . Rating the quality of evidence--publication Bias. J Clin Epidemiol 2011;64:1277-82. 